\title{
Chronic dacryosialadenitis in HTLV I associated myelopathy
}

\author{
Luis Cartier R, Jose Luis Castillo C, Jose Gabriel Cea M, Roque Villagra C
}

\begin{abstract}
A prospective study was carried out on 48 patients with HTLV I associated myelopathy/tropical spastic paraparesis (HAM/TSP) to assess the association between this entity and Sjögren's syndrome. Fourteen patients $(29 \cdot 1 \%)$ had chronic dacryosialadenitis confirmed by a positive Schirmer's test and salivary gland biopsy. None of these patients had evidence of collagen disease and tests for $\mathrm{Ro}, \mathrm{La}$, and rheumatoid factor were negative except in one case. Therefore, the dacryosialadenitis could not be classified as either primary or secondary Sjögren's syndrome. Ten of the 14 patients $(71 \cdot 4 \%)$ had other systems (haematological, articular, dermatological, or respiratory) involved apart from the neurological and exocrine gland pathology.

The findings suggest that the dacryosialadenitis associated with HTLV I is a disease of viral origin distinct from Sjögren's syndrome.
\end{abstract}

(F Neurol Neurosurg Psychiatry 1995;58:244-246)

Keywords: HTLV I associated myelopathy; dacryosialadenitis; Sjögren's syndrome

Myelopathy/tropical spastic paraparesis (HAM/TSP) associated with HTIV I is a clearly defined entity. The association of HAM/TSP with leukaemia/lymphoma, polyneuropathy, myositis, arthritis, lymphoalveolitis, and uveitis has been reported. Sjögren's syndrome is another association that has been reported in a few cases $^{12}$ but there is no detailed description of this clinical picture in HAM/TSP. This is a prospective study attempting to define the frequency and clinical features of Sjögren's syndrome in a large population of patients positive for HTLV I.

\section{Patients and methods}

Forty eight patients with HAM/TSP were evaluated during 1993 in our department of neurology. The characteristics of the spastic paraparesis has been described elsewhere. ${ }^{3-5}$
All patients were submitted to CSF examination, EMG, and nerve conduction studies, trimodal evoked potentials, and myelography; in some cases, CT and MRI were performed to exclude other causes of the paraparesis. Tests for venereal disease and HIV were negative in all cases. Status for HTLV I was determined either by enzyme linked immunosorbent assay (ELISA) or particle agglutination (Serodia Fujirebio) and confirmed by indirect immunofluorescence with the antigen of MT-2 cells and by western blot. Eight patients were studied with the polymerase chain reaction (Primers SK110, SK111, SK112, SK188).

Xerophthalmia was detected by Schirmer's test in all patients. Absorption of less than 8 $\mathrm{mm}$ in both eyes was considered to be positive. The 14 positive patients were submitted to a biopsy of the minor salivary glands. After injecting local anaesthetic, three or more salivary glands were taken from the external border of the lower lip, dissected out, and fixed in neutral formol and/or Bouin's fluid. The histological study was carried out with haematoxylin and eosin and Gomori stain and the histochemical study for lymphocytes was performed with Dako quick staining mouse antihuman $T$ cells.

A full blood count was performed in all patients to identify abnormal lymphocytes. Also, lymphocytes from blood and CSF were typed. In the blood samples they were separated from heparinised blood on a Ficollhypaque gradient and from the CSF by direct centrifugation. Cell identification was carried out by indirect immunofluorescence with monoclonal antibodies CD3, CD4, CD8 and CD22. Receptors for IL-2 in T lymphocytes in blood and CSF were studied in eight patients.

Serum protein electrophoresis with a Trisbarbitone sodium buffer at $\mathrm{pH} 8 \cdot 8$ and radial immunodiffusion to measure the serum immunoglobulin concentration were performed in Merck agar. The IgG index was calculated in CSF and blood. Rheumatoid factor was measured by the latex method; extractable nuclear antibodies (Ro and $\mathrm{La}$ ) by a double diffusion technique with purified cow thymus, and human spleen antigens and antinuclear antibodies with rat's kidneys. 
Skin biopsies measuring $2 \mathrm{~cm} \times 0.5 \mathrm{~cm}$ were taken from patients with skin lesions, fixed in formol or Bouin's fluid, processed in paraffin and stained with haematoxylin and eosin and Gomori stain. Other studies included bronchial brushing performed in two cases and bronchial biopsy in one.

\section{Results}

Fourteen out of 48 patients (29.1\%) proved positive for Schirmer's test. This group was composed of 11 women and three men with an average age of 50.9 (range 32-71) years. All patients presented with a spastic paraparesis, generalised hyper-reflexia, usually including a jaw jerk; bilateral Babinski sign; neurogenic bladder in $78.2 \%$. Two out of 14 had superficial and deep sensory changes that were minor.

Four patients had had paraparesis for 10 to 15 years; four for between five and nine years, and six for less than five years. Of these 14 patients, seven could walk unaided, three needed help, two used wheelchairs, and two were bedridden. Three cases developed a paraplegia in flexion.

All patients had bilateral xerophthalmia defined by Schirmer's test. Only half of the patients had dryness or red eyes, including one case with xerophthalmia and mouth ulcers. These symptoms began after the onset of the spastic paraparesis. The biopsies of the small salivary glands showed pronounced lymphocytic infiltrations even in those patients who did not complain of any symptoms. On the Chisholm and Mason scale, ${ }^{6}$ five cases were defined as grade 4 , with more than one cluster of 50 or more lymphocytes per $4 \mathrm{~mm}^{2}$. Eight cases were defined as grade 3 with one lymphocytic cluster per $4 \mathrm{~mm}^{2}$ and two cases as grade 2 , with diffuse lymphocytic infiltration (figure). The lymphocyte infiltrates contained plasma cells. Also, there was some loss or disruption of the acini and ductal proliferation. Twelve patients showed a multifocal sialadenitis.

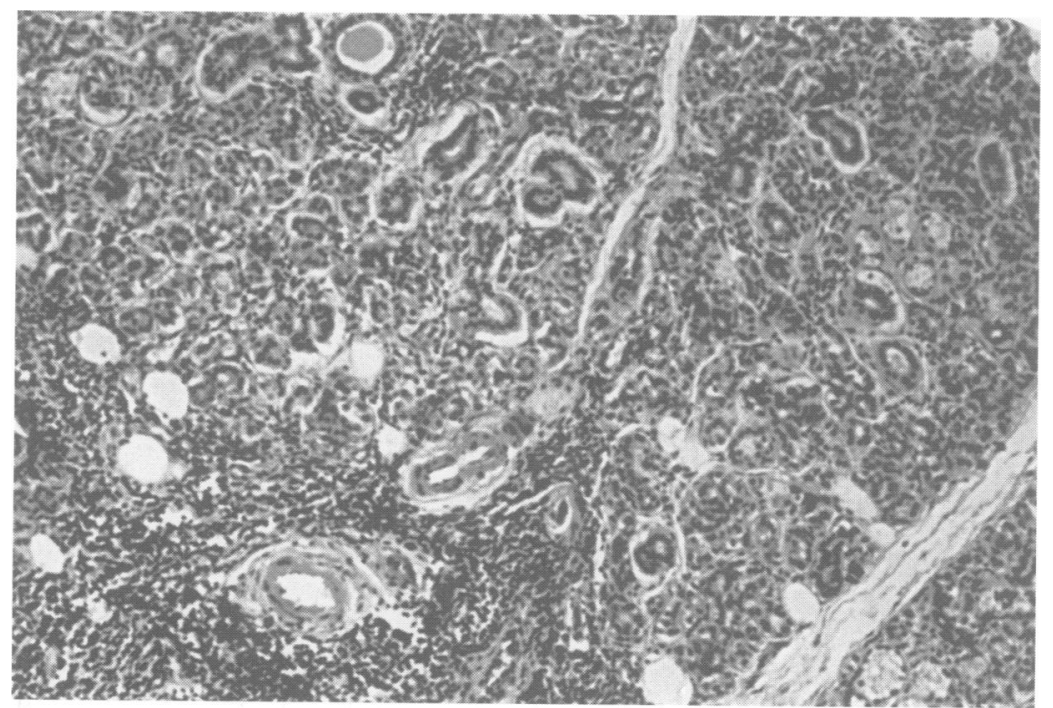

Salivary gland biopsy: lymphocytic infiltrates in the periductal and perivascular areas. Haematoxylin and eosin; originally $\times 150$.
In nine of the 14 patients electrophoresis of serum protein showed a rise in IgG greater than 1500. Studies of Ro and La, and antinuclear antibodies proved negative in all cases. Rheumatoid factor was strongly positive in only one case. An increase in CD3 (more than $62 \%$ ) and CD4 (more than $38 \%$ ) was found in the blood of $66 \%$ of patients. The lymphocytes in the CSF showed an increase in $\mathrm{CD} 3$ (more than $75 \%$ ) and CD4 (more than $45 \%$ ) in $88 \%$ of the patients. In the eight cases in which IL-2 receptors were studied lymphocytic activation occurred in four in blood or CSF. The CSF showed a pleocytosis (between six and 22 cells) in $58.3 \%$ of patients. Ten cases had an increase of the IgG index and 11 had oligoclonal bands.

Lobulated lymphomatous like lymphocytes were found in the full blood counts of nine patients, fluctuating from $1 \%$ to $14 \%$. Two patients showed associated lymphomatous changes, one developing a chronic and one an acute lymphoma.

Four patients had arthralgia and two had arthralgia and monarthritis in the ankle. Another case developed polyarthralgiae that preceded the spastic paraparesis. This was initially interpreted as a polyarthropaty in a mixed connective tissue disease, because she was the only one who had a rheumatoid factor of $1 / 5520$. In this patient, however, no articular changes apart from the arthralgiae were seen.

Four patients showed scaly erythematous papular cutaneous lesions and erythematous plaques. In these four and in two other patients (six out of 14) papular or macular erythematous rashes were seen, mainly on the limbs but also on the thorax and face. These either disappeared rapidly or persisted in a scaly form for long periods. The histological study of the skin lesions showed dermal infiltrations of $T$ lymphocytes with occasional epidermal and subepidermal infiltrations, which formed Pautrier's abscesses. There was perivascular infiltration, but no evidence of vasculitis.

In two patients the disease developed with persistent lymphoalveolitis. This diagnosis was made by bronchial brushing, and in one case, by biopsy, which showed T lymphocytic infiltration.

\section{Discussion}

The few patients with Sjögren's syndrome and HAM/TSP reported in the literature are all of negroid origin and, as far as we know, this clinical association has not been comprehensively described. ${ }^{127}$

Analysis of this series of 14 non-negroid patients shows that: $(a)$ in all patients the sicca symptoms seemed to start after the onset of the paraparesis and had no correlation with the time of evolution of HAM/TSP; (b) only half of the cases were symptomatic; the others were detected by the Schirmer's test; (c) $71 \%$ of patients had other systems involved, apart from the neurological and exocrine gland pathology (either haematologi- 
cal, articular, dermatological, or respiratory); (d) none of the patients had clinical evidence of collagen disease and all were negative for Ro and La; (e) the salivary gland biopsies showed multifocal sialadenitis.

According to clinical and pathological criteria $^{89}$ this series of patients could not be classified as having primary or secondary Sjögren's syndrome. The presence of dry eyes in our patients and the histological findings of the salivary glands provide two features in common with Sjögren's syndrome; however, no characteristic immunological response or any evidence of other autoimmune disease were found.

A special tropism of HTLV I for the lacrimal and salivary glands has been reported, demonstrating the presence of the protein 40 gen-tax ( 40 gen-tax) in the nucleus of these glands in transgenic mice that developed dacryosialadenitis. ${ }^{10}$ Also, the specific cytotoxic activity found in in vitro studies against the p40-tax could be related to the special lymphotropism in the salivary and lacrimal glands of patients with HAM/TSP." The presence of a high percentage of antibodies against p19 and p24 in some Sjögren's syndrome series has suggested a relation between retrovirus and dacryosialadenitis; however, in these series, HTLV I was not found..$^{12}{ }^{13}$ In our series the presence of HTLV I has been clearly shown.

These findings suggest that this dacryosialadenitis in patients with HAM/TSP has a viral origin. This pathology would be part of the multisystemic involvement found in HAM/TSP.

We thank Dr Eugenio Ramirez and colleagues from the Instituto de Salud Pública de Chile, Departamento de Virologia for their valuable help in the virology studies.

1 Gessain A, Barin F, Vernant JC, et al. Antibodies to human T-lymphotropic virus type-I in patients with human T-lymphotropic virus type-I in patients

2 Vernant J, Buisson G, Magdeleine J, et al. T-Lymphocyte alveolitis, tropical spastic paresis, and Sjögren syndrome. Lancet 1988;23:177.

3 Cartier L, Araya F, Castillo JL, et al. Progressive spastic paraparesis associated with human T-cell leukemia virus Type I (HTLV-I). Intern Med 1992;31:1257-61.

4 Cartier L, Araya F, Verdugo R. Progressive spastic paraparesis in Chile. In: Roman GC, Vernant JC, Osame $M$ eds. HTLV-I and the nervous system. New York: Alan R, 1989;51:167-73.

5 Cartier L, Mora C, Araya F, et al. HTLV-I positive spastic paraparesis in a temperate zone. Lancet 1989;i:556-7.

6 Chisholm D, Mason D. Labial salivary gland biopsy in Sjögren's disease. $f$ Clin Pathol 1968;21:656-60.

7 Vernant J, Buisson G, Sabersky G, et al. Can HTLV-I lead to immunological disease? Lancet 1987;ii:204.

8 Fox RI, Robinson CA, Curd JG, et al. Sjögren's Syndrome, proposed criteria for classification. Arthritis Rheum 1986;26:577-8

9 Lindstrom FD, Hellquist H, Olofsson J. Evaluation of lip salivary gland biopsy in 21 patients with primary Sjögren's syndrome. Clin Immunol Immunopathol 1987 45:156-65.

10 Green JE, Hinrichs SH, Vogel J, Jay G. Exocrinopathy resembling Sjögren's syndrome in HTLV-I tax transgenic mice. Nature 1989;341:72-4.

11 Kannagi M, Harada S, Maruyama I, et al. Predominant recognition of human $T$ cell leukemia virus type I (HTLV-I) pX gene products by human CD8 + cytotoxic $\mathrm{T}$ cell directed against HTLV-I infected cells. Int Immunol 1991;8:761-7.

12 Shattles W, Brookes S, Venables $\mathrm{P}$, et al. Expression of antigen reactive with a monoclonal antibody to HTLV-I antigen reactive with a monoclonal antibody to HTLV-1
P19 in salivary glands in Sjögren's syndrome. Clin Exp P19 in salivary glands in

13 Talal N, Dauphiné $M$, Dang $H$, et al. Detection of serum antibodies to retroviral proteins in patients with primary Sjögren's syndrome (autoimmune exocrinopathy) Arthritis Rheum 1990;33:774-81. 